\title{
Measurement of Single-Wall Nanotube Dispersion by Size Exclusion Chromatography ${ }^{\dagger}$
}

\author{
Barry J. Bauer,* Matthew L. Becker, Vardhan Bajpai, Jeffrey A. Fagan, Erik K. Hobbie, \\ Kalman Migler, Charles M. Guttman, and William R. Blair
}

Polymers Division, National Institute of Standards and Technology, Gaithersburg, Maryland 20899

Received: February 22, 2007; In Final Form: May 9, 2007

\begin{abstract}
Size exclusion chromatography (SEC) is shown to be an effective method to characterize single-wall carbon nanotube (SWNT) dispersions. SEC separates nanotube dispersions by size, and an on-line viscosity detector gives intrinsic viscosity as a function of hydrodynamic size, as is determined by universal calibration. The scaling contains information about the shape of the dispersed particles. This characterization method was tested on three representative dispersions, octadecylamine functionalization (SWNT-ODA) in tetrahydrofuran (THF), butyl group functionalization (SWNT-butyl) in THF, and DNA wrapping (SWNT/DNA) in aqueous solution. Significant differences between the dispersions were found. Small-angle neutron scattering (SANS) and atomic force microscopy (AFM) produced results consistent with the SEC method.
\end{abstract}

\section{Introduction}

Single-wall carbon nanotubes (SWNTs) have a variety of potential applications in materials due to their outstanding mechanical, electrical, optical, and thermal properties. ${ }^{1}$ However, current SWNT synthetic methods produce bundles of nanotubes comprised of tubes with a distribution of lengths, chiralities, and diameters that are often contaminated with non-SWNT carbon. Such mixtures are unsuitable for most applications and characterization methods without further processing. The dispersion of nanotubes in solution as individually separated nanotubes is necessary in order to achieve the goal of sorting and manipulating nanotubes by length and type and preparing high quality monodisperse samples. Monodisperse samples are also required in order to properly characterize the optical, thermal, and electrical properties of nanotube-based materials. Several schemes have been developed to promote SWNT dispersion, which have demonstrated the ability to form stable suspensions that do not settle out over long time periods. ${ }^{2}$ However, this nonsettling behavior is only a macroscopic measure of dispersion, and large aggregates of nanotubes may be present. Methods are necessary to assess the quality of the dispersions; for example, it must be known if the original bundles/ropes of nanotubes and ropes have broken down into isolated nanotubes. The nature of the dispersion, and our ability to characterize the dispersion on a size scale comparable to the SWNTs, is thus of great importance. We will present measurements of three different SWNT dispersions as measured by chromatographic separation, microscopy, and scattering.

Several techniques have been used to measure SWNT dispersion, each with its own shortcomings. Spectroscopic methods such as absorption spectroscopy and Raman spectroscopy are only weakly effected by aggregation and do not provide a quantitative measure. ${ }^{3-5}$ Fluorescence spectroscopy does detect semiconducting SWNTs that are not in ropes or tight bundles, but it is insensitive to the presence of metallic nanotubes. ${ }^{5}$ Scattering methods such as small-angle X-ray, ${ }^{6,7}$ neutron, ${ }^{8-13}$ and light scattering can detect aggregation, but these methods

\footnotetext{
† Part of the special issue "Richard E. Smalley Memorial Issue".

* To whom correspondence should be addressed. E-mail: barry.bauer@nist.gov.
}

are difficult to interpret when the solution contains significant heterogeneity; further, they are biased to the large (aggregated) fractions. Atomic force, scanning, and transmission microscopy (AFM, SEM, and TEM) ${ }^{4}$ only measure a small quantity of nanotubes and may be prone to biasing that would occur upon sample preparation if not all tube types are equally deposited in the visible field. Clearly, there is a need for a quantitative method for characterization of nanotube dispersions.

Size exclusion chromatography (SEC) is an effective method to characterize SWNT dispersions; it takes advantage of the size polydispersity inherent in nanotube samples. SEC has been reported for a variety of SWNT dispersions, ${ }^{14-23}$ giving confidence that this method can be applied generally. We have made measurements of the scaling relationship between the intrinsic viscosity $[\eta]$, as determined by on-line detectors, and the hydrodynamic volume $V_{\mathrm{H}}$, as determined by the universal calibration method. ${ }^{24}$ The shape of the nanodispersion (for example tubes, spheres, coils, or branched aggregates) can be discerned from this scaling relationship, thus providing information on the extent of dispersion as individual nanotubes. This characterization method is applied to three representative dispersion methods, octadecylamine attachment to acid-treated SWNTs (SWNT-ODA), butyl grafting through free-radical mechanisms (SWNT-butyl) (both dispersed in THF), and DNA wrapping in aqueous solution (SWNT/DNA). Significant differences between the dispersions were found. Small-angle neutron scattering (SANS) and atomic force microscopy (AFM) are independent measurement methods that produce results consistent with the SEC method.

The scaling is based on the Mark-Houwink-Sakurada $(\mathrm{MHS})^{24}$ equation

$$
[\eta]=K M^{a}
$$

which relates the intrinsic viscosity $[\eta]=\left(\eta(\phi)-\eta_{\mathrm{s}}\right) / \eta_{\mathrm{s}} \phi$ equation to $M$ through the MHS parameter $a$, which varies with extension and branching of the polymer or nanoparticle. The $\eta(\phi)$ is the viscosity of a solution with nanotube volume fraction $\phi$, and $\eta_{\mathrm{s}}$ is the viscosity of the solvent. Table 1 lists the MHS values of $a$ for six different structures ranging from rigid rods $(a=2)$ to dense spheres $(a=0)$. 
TABLE 1: Power Law Relationships for Ideal Structures (Top Six Lines) and Measured SWNT Dispersions (Bottom Three Lines) $)^{13,26,27}$

\begin{tabular}{lll}
\hline structure/dispersion method & $\begin{array}{c}\text { viscosity } \\
\text { power law } \\
{[\eta] \propto M^{a}}\end{array}$ & $\begin{array}{l}\text { scattering } \\
\text { power law } \\
I(q) \propto q^{-\alpha}\end{array}$ \\
\hline rigid rod & 2 & 1 \\
flexible rod & $0.8-2$ & $1-1.67$ \\
self-avoiding random walk & 0.8 & 1.67 \\
Gaussian random walk & 0.5 & 2 \\
branched & $<0.5$ & $2-3$ \\
dense sphere & 0 & 4 \\
SWNT-ODA & $0.15 \pm 0.03$ & $3.9 \pm 0.5$ \\
SWNT-butyl & $0.37 \pm 0.05$ & $2.5 \pm 0.2$ \\
SWNT-DNA & $1.14 \pm 0.20$ & $1.5 \pm 0.1$
\end{tabular}

Standard SEC methods developed for polymer solutions have been applied to the case of nanotube dispersions. In SEC, a porous column packing material is used to separate dissolved polymer molecules by size. The polymers partition between the flowing mobile phase and stationary phase that is incorporated within the pores of the column. The smaller polymers have a larger partitioning coefficient inside of the pores than that of the larger ones. This results in the larger polymers spending a larger fraction of time in the mobile phase, hence, eluting from the column before the smaller ones. ${ }^{24}$

The partitioning of the polymers will depend on their characteristic volume, which is commonly associated with their hydrodynamic volume. Universal calibration ${ }^{25}$ is a technique that assumes that elution time is a function of the hydrodynamic volume alone. Therefore, a column can be calibrated with a set of polymers having known hydrodynamic volumes and then used to measure other polymers or nanoparticles with unknown sizes and shapes.

SEC with universal calibration is used to measure the MHS parameter of unknown polymers by calibration of a column with a known polymer and by measuring the $[\eta]$ on line by employing a viscosity detector along with a concentration detector such as refractive index or ultraviolet absorption. ${ }^{24}$ When properly calibrated, $[\eta]$ at any elution time can be compared with the hydrodynamic volume from the calibration step. The hydrodynamic volume, $V_{\mathrm{H}}$, is related to the $[\eta]$ and molecular mass through a power law relationship

$$
V_{\mathrm{H}}=[\eta] M=K M^{a+1}
$$

Thus, a plot of measured on-line $[\eta]$ as a function of $V_{\mathrm{H}}$ from a calibrated column on a $\log -\log$ scale has a slope of $a /(a+1)$. By applying the strategy described above to the case of nanotube dispersions, the MHS parameter can be measured to determine the nature of the dispersion.

SANS can provide complementary information to estimate the nature of SWNT dispersion. The scattering from dispersed objects provides information on the size and shape of the object. The angle of scattering, $\theta$, and the neutron wavelength, $\lambda$, define the scattering parameter, $q=4 \pi \sin (\theta / 2) / \lambda$. A relative size scale, $d$, is probed by the $q$ value as $d=2 \pi / q$. If the value of $d$ is considerably smaller than the size of whole object, then the size itself cannot be determined accurately. However, the scattering in this region follows a power law, $I(q) \propto q^{-\alpha}$, which can be used to gain information on the density distribution of the internal structure of the object. Table 1 lists several objects and their associated power laws. ${ }^{26,27}$ The SANS power laws vary from 1 to 4 going from rods to spheres. This covers the same range of the viscosity power laws and can provide equivalent information on the nature of the dispersion.

\section{Experimental Section}

SWNTs were obtained from Carbon Nanotechnologies, Inc. ${ }^{28}$ The butyl grafting reaction (SWNT-butyl) was carried out according to the procedure of Billups et al. ${ }^{29}$ and was reported previously. ${ }^{13}$ The nanotubes were sonicated in benzene for 30 min using a bath sonicator. Butyl iodide, $\mathrm{C}_{4} \mathrm{H}_{9} \mathrm{I}$ (Aldrich Chemical, 99\%) was added along with benzoyl peroxide, and the mixture was refluxed for $24 \mathrm{~h}$. The reaction product was poured into methanol and centrifuged to remove solids. The solids were dispersed, precipitated, and centrifuged five times to remove unreacted material and dried in vacuum. The raw material was extracted with tetrahydrofuran (THF) and the soluble components concentrated to approximately $0.1 \mathrm{mg} / \mathrm{mL}$ for SEC.

The octadecylamine-grafted samples (SWNT-ODA) were prepared according to the standard procedure. ${ }^{14}$ The SWNTs were sonicated in a $3: 1$ mixture of $\mathrm{H}_{2} \mathrm{SO}_{4} /\left(70 \% \quad \mathrm{HNO}_{3}\right)$ at $60{ }^{\circ} \mathrm{C}$ for $120 \mathrm{~min}$ and purified by repeatedly washing with water. The dried SWNT was heated with octadecylamine (ODA) at $5 \%$ by mass at $110{ }^{\circ} \mathrm{C}$ for $50 \mathrm{~h}$. Unreacted ODA was removed by repeated washing with ethanol. The raw material was extracted with THF and the soluble components concentrated to approximately $0.1 \mathrm{mg} / \mathrm{mL}$ for SEC.

The aqueous DNA dispersions (SWNT/DNA) were prepared according to the procedure of Zheng et al. ${ }^{19,30,31}$ The SWNT was mixed with DNA containing 30 base pairs of alternating guanine and thymine in a $0.1 \mathrm{M} \mathrm{NaCl}$ aqueous salt solution with $1 \mathrm{mg}$ of DNA, $1 \mathrm{mg}$ of SWNT, and $1 \mathrm{~mL}$ of a $\mathrm{NaCl}$ solution. Dispersion was induced by sonication, and insoluble material was removed through centrifugation, resulting in a SWNT concentration of about $0.4 \mathrm{mg} / \mathrm{mL}$.

Size exclusion chromatography was performed on a Waters Alliance GPCV 2000 instrument using refractive index and viscosity detectors. The SWNT-butyl and SWNT-ODA samples were run at $1.0 \mathrm{~mL} / \mathrm{min}$ in THF using a Waters Styragel HMW 7E column. The SWNT/DNA analytical sample was run in $0.2 \mathrm{M} \mathrm{NaCl}$ and $0.04 \mathrm{M}$ TRIS at $\mathrm{pH}=7.0$ at $0.5 \mathrm{~mL} / \mathrm{min}$ using a SepaxCNT (SEC-1000 + SEC-300) column set. The SWNT/DNA preparatory run used a SepaxCNT (SEC-2000, SEC-1000 + SEC-300) column set, and fractions were collected every 2 min. All columns were calibrated in THF with narrow PS standards in THF. All samples were passed through a $0.45 \mu \mathrm{m}$ filter before injection.

Small-angle neutron scattering was performed on the NG7 $30 \mathrm{~m}$ instrument at the National Institute of Standards and Technology Center for Neutron Research (NCNR). The number of counts was corrected for detector sensitivity, background scattering, and empty cell effects. Absolute scattering intensities were calculated by comparison to the empty beam intensity, and data were corrected using the experimental values for sample transmissions. Incoherent scattering was removed by subtraction of a solvent blank.

Tapping-mode atomic force microscopy measurements were conducted in air using a Nanoscope IV system (Digital Instruments) operated under ambient conditions with standard silicon tips (NanoDevices Metrology Probes; L, $125 \mu \mathrm{m}$; normal spring constant, $40 \mathrm{~N} / \mathrm{m}$; resonance frequency, $280-330 \mathrm{kHz}$ ). Individual populations of acid-refluxed SWNT-ODA, DNAwrapped SWNT/DNA, and butyl-grafted nanotubes (SWNTbutyl) were prepared as described previously. Briefly, the centrifuged solutions of SWNT-ODA and SWNT/DNA were diluted $100 \times$ in water $\left(18 \mathrm{M} \Omega \mathrm{cm}^{-1}\right)$ prior to being deposited ( $2 \mathrm{~mL}$ ) onto plasma-cleansed $\mathrm{Si}[1,1,1]$ wafers or freshly cleaved mica. After being allowed to dry, any residual salt was washed 


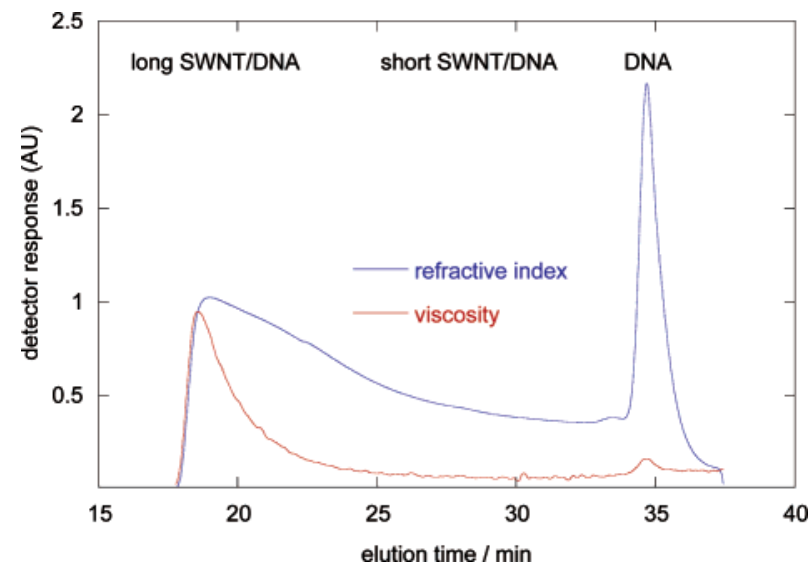

Figure 1. Normalized response from viscosity and refractive index detectors as a function of elution time in the SEC of SWNT/DNA.

away by a water deposition/wicking procedure (2-3 times) to afford clear imaging conditions. SWNT-butyl was prepared via $100 \mathrm{rad} / \mathrm{sec}$ spin coating from chloroform solvent on a $\mathrm{Si}$ wafer with no washing and air drying.

The relative uncertainties reported are one standard deviation based on the goodness of the fit or from multiple runs. Total combined uncertainties from all external sources are not reported, as comparisons are made with data obtained under the same conditions. In cases where the limits are smaller than the plotted symbols, the limits are left out for clarity.

\section{Results and Discussion}

Figure 1 shows the response of viscosity and refractive index detectors for the SEC separation of a typical aqueous SWNT/ DNA dispersion. The refractive index detector is sensitive to the concentration in mass per volume and is insensitive to molar mass for large macromolecules. The viscosity detector measures the pressure drop across a capillary, producing a signal that is dependent on both the concentration and the size of the dispersed material. The response of the two detectors is clearly different, with the viscosity detector producing a signal that drops far more rapidly than the refractive index detector. SEC separates by size, with the larger dispersed materials eluting before the smaller ones. The unattached DNA elutes near the end of the separation due to its small molar mass of less than $10,000 \mathrm{~g} / \mathrm{mol}$. A ratio of the viscosity detector to the refractive index detector produces $[\eta]$ when calibrated for refractive index increment and capillary size.

Figure 2 is a plot of $[\eta]$ measured using on-line SEC with columns calibrated with narrow polystyrene (PS) standards. The calibration used a literature value of MHS parameters of $K=$ $0.011 \mathrm{~mL} / \mathrm{g}$ and $a=0.725 .^{32}$ The $a$ value from a broad PS mixture is $0.73 \pm 0.02$, which is in agreement with the literature value used in the calibration. The three SWNT dispersions have considerably different slopes in the plots, and the extracted values of $a$ are listed in Table 1. The SWNT-ODA and SWNT-butyl dispersions produced weak viscometer signals, producing low $[\eta]$ values, and hence, they have a more compact structure. The SWNT/DNA gave stronger viscometer response, indicating a larger structure.

Table 1 lists the MHS values calculated from the slopes in Figure 2. The SWNT-ODA dispersion produces a value of $a=0.15 \pm 0.03$, indicating a very dense structure close to that of a solid sphere. The SWNT-butyl produces a value of $a=$ $0.37 \pm 0.05$, which falls in the range of branched structures. While it cannot be determined whether the branches are rods or flexible chains, the low exponent size is inconsistent with

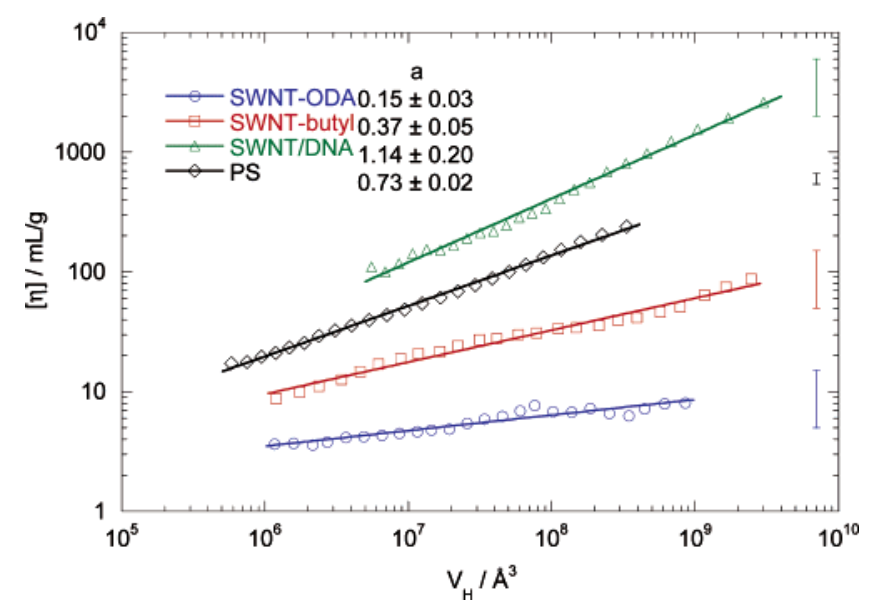

Figure 2. Intrinsic viscosity measured on line by SEC. $V_{\mathrm{H}}$ is from universal calibration using columns calibrated with PS standards.

unbranched chains or rods. The SWNT/DNA produces a value of $a=1.14 \pm 0.20$, which falls in the range of flexible rods. Mixed structures such as individual rigid rods and lightly branched rods would produce MHS parameters in between those of the components, but such a combination would be likely to cause curvature in the lines of Figure 2 if the SEC separation process separates the components differently.

AFM images provide complementary information. Figure 3 shows AFM images of SWNTs dispersed by the three methods. SWNT-ODA shows irregular spheres with high polydispersity (A), SWNT-butyl shows branched clusters of many tubes (B), and SWNT/DNA shows individual stiff rods (C). The micrographs $(5 \mu \mathrm{m} \times 5 \mu \mathrm{m})$ are representative of each sample type and clearly place each in a different class. This qualitative observation is consistent with the results obtained through SEC.

The uniformity of the SWNT/DNA dispersion allows for a statistical measurement of SWNT size for samples eluting at various times. The SEC separation of SWNT/DNA was repeated, and fractions were collected for AFM analysis on a column set that was calibrated with polystyrene standards in THF. Figure 4 shows AFM images of four fractions taken at increasing times. Between 50 and 100 SWNTs were measured, and size distributions were calculated from the lengths in the image.

Figure 5 is a plot of the average length from AFM as a function of the elution time of the collection. The molecular mass of PS standards that eluted in the same time range as the SWNT/DNA fractions is also plotted. The thick lines through the data points are fits of an exponential to the data. The goodness of the fits is a result of the broad pore size distribution of the column packing with both the PS and the SWNT/DNA being in the linear range of the column. The fit of the PS molar mass and the SWNT/DNA length are highly correlated, with a relationship given by eq 3

$$
L(\mathrm{~nm})=0.00573 M(\mathrm{~g} / \mathrm{mol})^{0.806}
$$

This relationship can be compared to the results shown in Figure 2 and Table 1 that have been calculated using universal calibration. The AFM length is proportional to molar mass if the diameter size distribution of the SWNTs is not significantly changed by SEC fractionation. ${ }^{19}$ Therefore, the power law exponent of 0.806 from eq 3 also relates the PS and SWNT/ DNA molar masses. This power law can be calculated from 

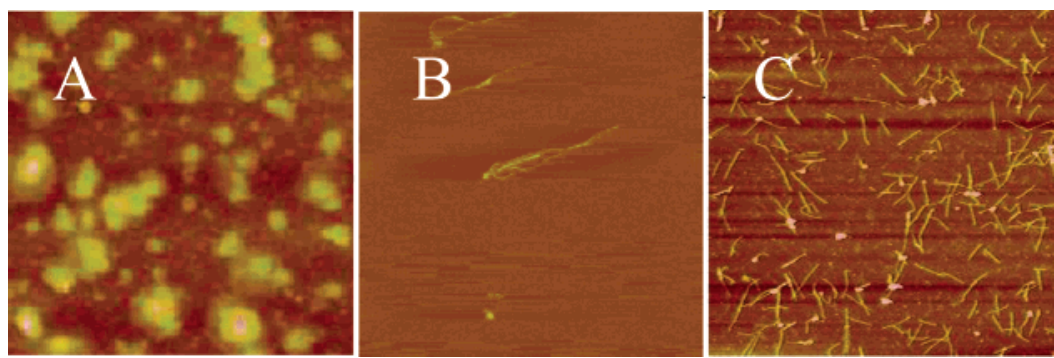

Figure 3. Representative AFM micrographs $(5 \times 5 \mu \mathrm{m})$ depicting the results from the three preparative methods described in the text, SWNTODA (A), SWNT-butyl (B), and SWNT/DNA (C).

\section{fraction (minutes)}

37
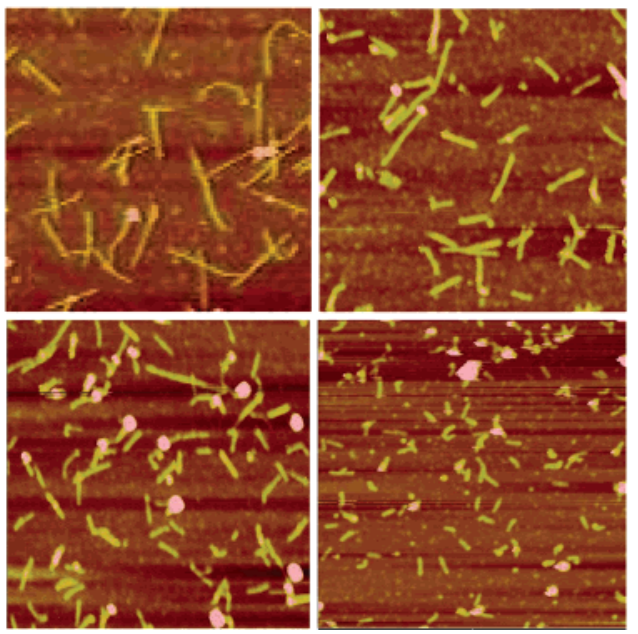

Figure 4. Representative AFM micrographs $(2.5 \times 2.5 \mu \mathrm{m})$ depicting the SEC fractions taken at various times from SWNT/DNA.

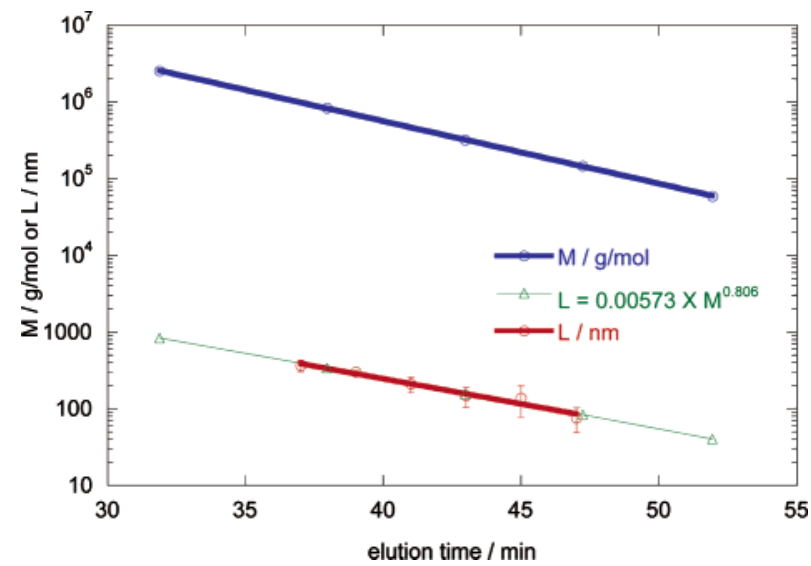

Figure 5. Polystyrene molar mass and SWNT/DNA length from AFM as a function of SEC elution time.

the MHS coefficient by the use of universal calibration. If $M[\eta]=K M^{a+1}$ is the same for both PS and SWNT/DNA, then

$$
M_{\mathrm{PS}}{ }^{0.73+1} \propto M_{\mathrm{SWNT} /} \mathrm{DNA}^{1.14+1} \text { or } L \propto M_{\mathrm{PS}}{ }^{0.808}
$$

The agreement of these two different methods of exponent calculation provides additional evidence that universal calibration applies to these dispersions. Additionally, eq 3 may prove to be a useful estimate of SWNT/DNA length from fractions collected on a SEC column calibrated by PS standards.

Figure 6 shows the SANS results from the SWNT-butyl, the SWNT-ODA, and the SWNT/DNA dispersions. The SWNT-butyl and SWNT-ODA dispersions show power law behavior over a range of $q$. Table 1 indicates the power law

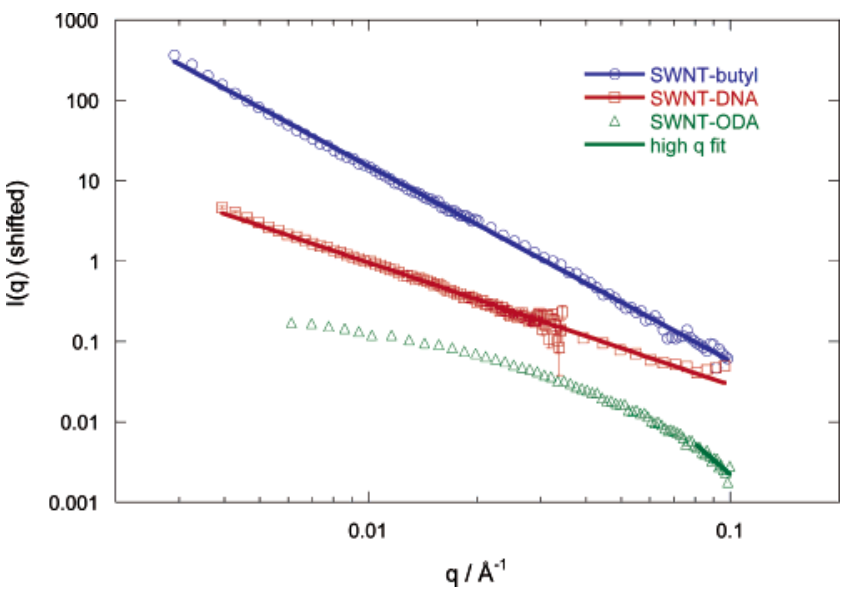

Figure 6. SANS from SWNT dispersions. SWNT-butyl has a wide power law region of $2.5 \pm 0.2, \mathrm{SWNT} / \mathrm{DNA}$ has a wide power law region of $1.5 \pm 0.1$, and SWNT-ODA has a curved region with a power law of $3.9 \pm 0.5$ at the highest $q$ region measured.

exponents, $\alpha$, expected from typical structures, in a fashion analogous to the viscosity power laws. The SWNT-butyl has an extended power law region of $2.4 \pm 0.2,{ }^{13}$ and the SWNT/ DNA shows an extended power law region of $1.5 \pm 0.1$. The SWNT-ODA has a broad curvature typical of large polydispersity, with a slope of $3.9 \pm 0.5$ at the highest $q$, as reported in Table 1.

The SEC with on-line detection is clearly able to measure the large differences between the three dispersions, as seen in the summary of results at the bottom of Table 1. SEC indicates that the SWNT-butyl is a branched structure, and it indicates that the SWNT/DNA is a semiflexible rod, clearly stiffer than a self-avoiding walk but more flexible than a rigid rod. The SEC result from the SWNT-ODA shows that the eluent is a dense object, close to spherical in nature, and most likely comprised predominately of carbonaceous masses. These results are consistent with those seen in the AFM images. SANS provides a complimentary result, as shown in Table 1.

The choice of the three dispersion methods used in this study was intended to represent a variety of typical dispersion schemes. SEC separations of SWNT-ODA have been reported previously. ${ }^{14,20,22}$ Reports have found an initial fraction containing dispersed SWNTs followed by fractions of primarily carbonaceous impurities, and other reports using acid-purified SWNTs also found considerable carbonaceous impurities. ${ }^{20,22}$ Our measurements are consistent with the presence of a significant amount of this carbonaceous impurity. A published report of SEC of SWNT/DNA dispersions describes good dispersions, which is consistent with our findings. ${ }^{19,30,31}$ The SWNT-butyl SANS and AFM results have been previously reported. ${ }^{13}$

Additional measurements of SWNT dispersion have recently been made on nanotube dispersions in a solid matrix. ${ }^{33} \mathrm{SWNT} /$ 
DNA aqueous dispersions were prepared and mixed with poly(acrylic acid) solutions at controlled $\mathrm{pH}$. While chromatographic dispersion measurements are not possible for solid samples, SANS can be used to access the quality of the dispersions. A power law of -1 was found for a dispersion prepared at $\mathrm{pH}=$ 2.2 , while a dispersion prepared at $\mathrm{pH}=4.1 \mathrm{had}$ a power law of -2.5 . The -1 power law is characteristic of singly dispersed SWNTs.

In summary, a method for quantifying dispersion using SEC with concentration and viscosity detection has been demonstrated with three dispersions of SWNTs. Chromatography is becoming an important method of purifying and sorting SWNT types. The use of size-sensitive on-line detection can provide important information on the nature of the dispersion. All three of the dispersions described in this article formed dark-black liquids even after centrifugation and passing through a $0.45 \mu \mathrm{m}$ syringe filter. SWNT dispersions that can pass through a SEC column may still have components of clustered nanotubes or non-SWNT carbon.

Acknowledgment. The support of Derek Ho, Charles Glinka, and Paul Butler of NCNR in aiding in collection of the neutron scattering data and of Ming Zheng for providing one of the samples used for SANS of SWNT/DNA is gratefully acknowledged. We acknowledge the support of the National Institute of Standards and Technology, U.S. Department of Commerce, in providing the neutron research facilities used in this work.

\section{References and Notes}

(1) Baughman, R. H.; Zakhidov, A. A.; de Heer, W. A. Science 2002, 297, 787-792.

(2) Haddon, R. C.; Sippel, J.; Rinzler, A. G.; Papadimitrakopoulos, F. MRS Bull. 2004, 29, 252-259.

(3) O'Connell, M. J.; Sivaram, S.; Doorn, S. K. Phys. Rev. B 2004, 69, 235415/1-235415/15

(4) Belin, T.; Epron, R. Mater. Sci. Eng., B 2005, 119, 105-118.

(5) Dresselhaus, M. S.; Samsonidze, G. G.; Chou, S. G.; Dresselhaus, G.; Jiang, J.; Saito, R.; Jori, A. Physica E 2005, 29, 443-446.

(6) Schaefer, D.; Brown, J. M.; Anderson, D. P.; Zhao, J.; Chokalingam,

K.; Tomlin, D.; Ilavsky, J. J. Appl. Crystallogr. 2003, 36, 553-557.

(7) Schaefer, D. W.; Zhao, J.; Brown, J. M.; Anderson, D. P.; Tomlin,

D. W. Chem. Phys. Lett. 2003, 375, 369-375.

(8) Rols, S.; Anglaret, E.; Sauvajol, J. L.; Coddens, G.; Dianoux, A. J. Appl. Phys. A 1999, 69, 591-596.

(9) Wang, H.; Zhou, W.; Ho, D. L.; Winey, K. I.; Fischer, J. E.; Glinka, C. J.; Hobbie, E. K. Nano Lett. 2004, 4, 1789-1793.

(10) Yurekli, K.; Mitchell, C. A.; Krishnamoorti, R. J. Am. Chem. Soc. 2004, 126, 9902-9903.
(11) Zhou, W.; Islam, M. F.; Wang, H.; Ho, D. L.; Yodh, A. G.; Winey, K. I.; Fischer, J. E. Chem. Phys. Lett. 2004, 384, 185-189.

(12) Hough, L. A.; Islam, M. F.; Hammouda, B.; Yodh, A. G.; Heiney, P. A. Nano Lett. 2006, 6, 313-317.

(13) Bauer, B. J.; Hobbie, E. K.; Becker, M. L. Macromolecules 2006, $39,2637-2642$

(14) Chattopadhyay, D.; Lastella, S.; Kim, S.; Papadimitrakopoulos, F. J. Am. Chem. Soc. 2002, 124, 728-729.

(15) Duesberg, G. S.; Muster, J.; Krstic, V.; Burghard, M.; Roth, S. Appl. Phys. A 1998, 67, 117-119.

(16) Duesberg, G. S.; Blau, W.; Byrne, H. J.; Muster, J.; Burghard, M.; Roth, S. Synth. Met. 1999, 103, 2484-2485.

(17) Farkas, E.; Anderson, M. E.; Chen, Z. H.; Rinzler, A. G. Chem. Phys. Lett. 2002, 363, 111-116.

(18) Holzinger, M.; Hirsch, A.; Bernier, P.; Duesberg, G. S.; Burghard, M. Appl. Phys. A 2000, 70, 599-602.

(19) Huang, X. Y.; McLean, R. S.; Zheng, M. Anal. Chem. 2005, 77, $6225-6228$

(20) Niyogi, S.; Hu, H.; Hamon, M. A.; Bhowmik, P.; Zhao, B.; Rozenzhak, S. M.; Chen, J.; Itkis, M. E.; Meier, M. S.; Haddon, R. C. J. Am. Chem. Soc. 2001, 123, 733-734.

(21) Yang, Y. L.; Xie, L. M.; Chen, Z.; Liu, M. H.; Zhu, T.; Liu, Z. F. Synth. Met. 2005, 155, 455-460.

(22) Zhao, B.; Hu, H.; Niyogi, S.; Itkis, M. E.; Hamon, M. A.; Bhowmik, P.; Meier, M. S.; Haddon, R. C. J. Am. Chem. Soc. 2001, 123, 1167311677.

(23) Heller, D. A.; Mayrhofer, R. M.; Baik, S.; Grinkova, Y. V.; Usrey, M. L.; Strano, M. S. J. Am. Chem. Soc. 2004, 126, 14567-14573.

(24) Mori, S.; Barth, H. G. Size Exclusion Chromatography; Springer: Berlin, Germany, 1999.

(25) Grubisic, Z.; Rempp, P.; Benoit, H. J. Polym. Sci., Polym. Lett. Ed. 1967, 5, 753-759.

(26) Higgins, J. S.; Benoit, H. C. Polymers and Neutron Scattering; Clarendon Press: Oxford, U.K., 1997.

(27) Roe, R. J. Methods of X-Ray and Neutron Scattering in Polymer Science; Oxford University Press: New York, 2000.

(28) Certain commercial equipment and materials are identified in this paper in order to adequately specify the experimental procedure. In no case does such identification imply recommendation by NIST nor does it imply that the material or equipment identified is necessarily the best available for this purpose.

(29) Ying, Y. M.; Saini, R. K.; Liang, F.; Sadana, A. K.; Billups, W. E. Org. Lett. 2003, 5, 1471-1473.

(30) Zheng, M.; Jagota, A.; Strano, M. S.; Santos, A. P.; Barone, P.; Chou, S. G.; Diner, B. A.; Dresselhaus, M. S.; McLean, R. S.; Onoa, G. B.; Samsonidze, G. G.; Semke, E. D.; Usrey, M.; Walls, D. J. Science 2003 , $302,1545-1548$

(31) Zheng, M.; Jagota, A.; Semke, E. D.; Diner, B. A.; McLean, R. S.; Lustig, S. R.; Richardson, R. E.; Tassi, N. G. Nat. Mater. 2003, 2, 338342 .

(32) Spatorico, A. L.; Coulter, B. J. Polym. Sci., Polym. Phys. Ed. 1973, $11,1139-1150$

(33) Fagan, J. A.; Landi, B. J.; Mandelbaum, I.; Simpson, J. R.; Bajpai, V.; Bauer, B. J.; Migler, K.; Walker, A. R. H.; Raffaelle, R.; Hobbie, E. K. J. Phys. Chem. B 2006, 110, 23801-23805. 